\title{
STABILITY OF ISOMETRIES
}

\author{
BY
}

PETER M. GRUBER

\begin{abstract}
A map $T: E \rightarrow F(E, F$ Banach spaces) is called an $\varepsilon$-isometry if $|\|T(x)-T(y)\|-\|x-y\||<\varepsilon$ whenever $x, y \in E$. Hyers and Ulam raised the problem whether there exists a constant $\kappa$, depending only on $E$, $F$, such that for every surjective $\varepsilon$-isometry $T: E \rightarrow F$ there exists an isometry $I: E \rightarrow F$ with $\|T(x)-I(x)\|<\kappa \varepsilon$ for every $x \in E$. It is shown that, whenever this problem has a solution for $E, F$, one can assume $\kappa<5$. In particular this holds true in the finite dimensional case.
\end{abstract}

1. Introduction. Let $E$ and $F$ be (real) Banach spaces and $\varepsilon>0$. Following Hyers and Ulam [11] a mapping $T: E \rightarrow F$ is called an $\varepsilon$-isometry if $\| T(x)$ $-T(y)\|-\| x-y \| \mid \leqslant \varepsilon$ whenever $x, y \in E$. (We denote the norms in $E$ and $F$ by the same symbol \|\| .) Hyers and Ulam [10] raised the problem whether there exists a constant $\kappa=\kappa(E, F)>0$ depending only on $E$ and $F$ with the following property: For each $\varepsilon>0$ and surjective $\varepsilon$-isometry $T$ : $E \rightarrow F$ there exists an isometry $I: E \rightarrow F$ with $\|T(x)-I(x)\| \leqslant \kappa \varepsilon$ for each $x \in E$. The assumption that $T$ be surjective is necessary (cf. Hyers and Ulam [11] and Theorem 2 below).

The question of Hyers and Ulam belongs to the following general stability problem: Suppose a mathematical object satisfies a certain property approximately. Is it then possible to approximate this object by objects, satisfying the property exactly? This problem is of particular interest in probability theory and in the case of functional equations of different types. A general discussion of this problem and a survey of some older results is contained in the interesting papers of D. G. Bourgin [5] and Ulam [15].

We return to the original problem. Hyers and Ulam [12] showed that the answer is affirmative in case $E=F$ is a Hilbert space. A possible value for $\kappa$ is 10. D. G. Bourgin [3] gave a positive answer whenever $F$ belongs to a class of uniformly convex Banach spaces including the $L_{p}[0,1]$ spaces for $1<p<$ $\infty$. Here one can take $\kappa=12$. A subsequent paper of Hyers and Ulam [13] gave a positive answer for the case that $E$ and $F$ are the Banach spaces of real

Received by the editors August 30, 1977.

AMS (MOS) subject classifications (1970). Primary 39A20, 41A45, 46B99; Secondary 41A65, 47A65, 47H99, 52A20.

Key words and phrases. Approximate isometry, isometry, stability of isometries, Banach space, normed space, finite dimensional normed space, stability of functional equations, stability, Löner's ellipsoid. 
continuous functions on compact Hausdorff spaces with the supremum norm, provided $T$ is a homeomorphism. In particular one can assume $\kappa=21$. This study was continued by D. G. Bourgin [4] who showed that several assumptions could be weakened. Papers [13] and [4] provide a significant generalization of the classical theorem of Banach and Stone (cf. [9, p. 115]). D. G. Bourgin [4] also investigates questions belonging to the more general problem mentioned above. Some time ago his son R. D. Bourgin [6] reconsidered the problem of Hyers and Ulam and investigated primarily the finite dimensional case. He showed that in this case the answer is almost positive if one assumes (1) $E=F$ and (2) the set of extreme boundary points of the unit ball is totally disconnected. Condition (2) is certainly satisfied if the unit ball is a polyhedron. If $E=F$ is a $d$-dimensional $l_{1}$-space, one can put $\kappa=100 d^{3}$ $-50 d^{2}+25 d+3$. Besides these results R. D. Bourgin proved some further results which are important in the infinite dimensional case. Some of these will be mentioned below.

In particular we show in $\$ 2$ the following. If the problem of Hyers and Ulam has a positive solution for a pair $E, F$ of Banach spaces, then one can assume $\kappa \leqslant 5$ (Theorem 1). In the proof theorems of Figiel [10] and R. D. Bourgin [6] are used. This result shows in particular that the values of $\kappa$ given above can be replaced by 5 throughout. In [6, p. 324], R. D. Bourgin states the conjecture that there exist separable Banach spaces, for which the answer to the problem is negative. Our result shows that it is not possible to confirm the conjecture in the way proposed by Bourgin. Generalizing a one-dimensional example of Hyers and Ulam [12] we prove the necessity of the assumption that $T$ be surjective (Theorem 2).

In $\$ 3$ the finite dimensional case is considered. It is shown that in this case the problem of Hyers and Ulam has a positive answer (Theorem 3). Tools for proving this are results of R. D. Bourgin [6], some properties of Löwner's minimal ellipsoid (cf. [7]), Brouwer's theorem on the invariance of domain (cf. $[1$, p. 396]) and the theorem of Mazur-Ulam (cf. [2, p. 160] and [9, p. 142]).

Let 0 denote the origin of $E$ and $F$ resp. $o(\cdot)$ stands for the well-known Landau symbol. dim, diam and bd denote dimension, diameter and boundary. $B$ is the solid unit ball of $E$.

\section{The general case.}

THEOREM 1. Let $E$ and $F$ be real normed spaces. Suppose that $\varepsilon \in \mathbf{R}^{+}, T$ : $E \rightarrow F$ is a surjective $\varepsilon$-isometry and that $I: E \rightarrow F$ is an isometry such that $T(p)=I(p)$ for some $p \in E$. If $\|T(x)-I(x)\|=o(\|x\|)$ as $\|x\| \rightarrow \infty$ uniformly, then $I$ is a surjective linear isometry and $\|T(x)-I(x)\|<5 \varepsilon$ for all $x \in E$. If in addition $T$ is continuous, then $\|T(x)-I(x)\|<3 \varepsilon$ for all $x \in E$.

RemarK. Suppose that (1) $E$ is Euclidean or (2) $E$ is isometric isomorphic 
to the space of continuous functions on an extremally disconnected compact Hausdorff space. Let $K$ be a subset of $E$ with diam $K=2$. If (1) holds, a theorem of Kirszbraun and Valentine (cf. [11, p. 169]) can be used to prove that $K$ is contained in a ball of radius $2^{1 / 2}$. If (2) holds a theorem of several authors (cf. [9, p. 123], [11, p. 170]) shows that $K$ is contained in a ball of radius 1 . These results yield improvements of the constants in Theorem 1: 3, 5 change to $3 \cdot 2^{-1 / 2}=212132 \ldots, 5 \cdot 2^{-1 / 2}=353553 \ldots$ in case (1) holds and to 15,25 in case (2) holds if $I$ is replaced by $I+q$ for an appropriate $q \in F$. Other improvements are possible.

Proof. At first we put together some tools. The first one is a theorem of Figiel [10]:

Let $M, N$ be real normed linear spaces and $R: M \rightarrow N$ an isometry with $R(0)=0$ such that $N$ is the closed linear hull of $R(M)$. Then there exists a linear map $S: N \rightarrow M$ of norm one for which $S \circ R=\mathrm{id}_{M}$.

Here $\mathrm{id}_{M}$ denotes the identical mapping of $M$ into itself. From the proof of Lemma 2.8 of R. D. Bourgin and P. Renz [6] we conclude:

Let $M$ be a real normed linear space, $\vartheta, \delta \in \mathbf{R}^{+}$and $R$ : $M \rightarrow M$ a $\vartheta$-isometry with $R(0)=0$. Then there exists a continuous map $S: M \rightarrow M$ with $\|R(x)-S(x)\|<\vartheta+\delta$ for all $x \in M$.

We now turn to the proof of Theorem 1 . Obviously we may assume

$$
T(0)=I(0)=0 \text {. }
$$

Our first goal is to show that

$I$ is surjective and linear.

Since $T$ is a surjective $\varepsilon$-isometry and $\|T(x)-I(x)\|=o(\|x\|)$ as $\|x\| \rightarrow \infty$ uniformly and hence $\|T(x)-I(x)\|=o(\|T(x)\|)$ as $\|T(x)\| \rightarrow \infty$ uniformly, $F$ is the closure of the linear hull of $I(E)$. This together with (1) shows the existence of a map

$$
J: F \rightarrow E, \quad \text { linear, with } J \circ I=\mathrm{id}_{E}
$$

and $\|J\|=1$. We now show

$$
J \text { is bijective. }
$$

Assume that $J$ is not injective. Because of the linearity of $J$ then there exists $y \in F \backslash\{0\}$ with $J(y)=0$. It follows $J(\lambda y)=0$ for all $\lambda \in \mathbf{R}^{+} . T$ is a surjective $\varepsilon$-isometry and $\|T(x)-I(x)\|=o(\|x\|)$ as $\|x\| \rightarrow \infty$ uniformly. Hence $\|T(x)-I(x)\|=o(\|T(x)\|)$ as $\|T(x)\| \rightarrow \infty$ uniformly. Therefore it is possible to choose for each $\lambda \in \mathbf{R}^{+}$a point $x_{\lambda} \in E$ such that $\left\|\lambda y-I\left(x_{\lambda}\right)\right\|$ 
$=o(\|\lambda y\|)=o(\lambda)$ as $\lambda \rightarrow \infty$. This together with the fact that $I$ is an isometry shows $\left\|x_{\lambda}\right\|=\left\|I\left(x_{\lambda}\right)\right\|=\|\lambda y\|+o(\lambda)=\lambda\|y\|+o(\lambda)$ as $\lambda \rightarrow \infty$. Combining these equalities, $J \circ I=\mathrm{id}_{E}$ and $\|J\|=1$ we have

$$
\begin{aligned}
0 & =\|J(\lambda y)\| \geqslant\left\|J\left(I\left(x_{\lambda}\right)\right)\right\|-\left\|J(\lambda y)-J\left(I\left(x_{\lambda}\right)\right)\right\| \\
& \geqslant\left\|x_{\lambda}\right\|-\left\|\lambda y-I\left(x_{\lambda}\right)\right\|=\lambda\|y\|+o(\lambda) \quad \text { as } \lambda \rightarrow \infty .
\end{aligned}
$$

This contradiction shows that $J$ is injective. Since $J \circ I=\mathrm{id}_{E}, J$ is surjective also. This proves (5). Now (3) follows from (4) and (5) $\left(I=J^{-1}\right)$. The next step is to show that

$$
I(x)=\lim _{n \rightarrow \infty} 2^{-n} T\left(2^{n} x\right) \text { for each } x \in E .
$$

Recall that $\|T(x)-I(x)\|=o(\|x\|)$ as $\|x\| \rightarrow \infty$ uniformly. From this we have for each $x \in E$ that $\left\|T\left(2^{n} x\right)-I\left(2^{n} x\right)\right\|=o\left(2^{n}\right)$ as $n \rightarrow \infty$ or $\left\|2^{-n} T\left(2^{n} x\right)-I(x)\right\|=o(1)$ as $n \rightarrow \infty$. This yields (6).

REMARK. In the proof of Theorem 1 we will use from now on only (2), the fact that $I$ is a linear, surjective isometry (cf. (3)), that $T$ is a surjective $\varepsilon$-isometry with $T(0)=0$ and (6).

The fact that $T$ is a surjective $\varepsilon$-isometry with $T(0)=0$ together with (3) and (6) gives

$$
\begin{aligned}
U & :=I^{-1} T: E \rightarrow E \text { is a surjective } \varepsilon \text {-isometry with } U(0)=0, \\
x & =\lim _{n \rightarrow \infty} 2^{-n} U\left(2^{n} x\right) \text { for each } x \in E .
\end{aligned}
$$

Now choose a map $U^{-1}: E \rightarrow E$ in the following way: $U^{-1}(0):=0$ and for $x \in E \backslash\{0\}$ let $U^{-1}(x)$ be any point $y \in E$ with $U(y)=x$. Obviously $U^{-1}$ has the following properties:

$$
\begin{gathered}
U^{-1}: E \rightarrow E \text { is an } \varepsilon \text {-isometry with } U^{-1}(0)=0, \\
U\left(U^{-1}(x)\right)=x \text { for each } x \in E .
\end{gathered}
$$

We show

$$
x=\lim _{n \rightarrow \infty} 2^{-n} U^{-1}\left(2^{n} x\right) \text { for each } x \in E .
$$

For $x \in E$ it follows from (7), (10) and (8) that

$$
\begin{aligned}
\left\|2^{-n} U^{-1}\left(2^{n} x\right)-x\right\| & =2^{-n}\left\|U^{-1}\left(2^{n} x\right)-2^{n} x\right\| \\
& \leqslant 2^{-n}\left(\left\|2^{n} x-U\left(2^{n} x\right)\right\|+\varepsilon\right)=\left\|x-2^{-n} U\left(2^{n} x\right)\right\|+2^{-n} \varepsilon \\
& \rightarrow 0 \text { as } n \rightarrow \infty .
\end{aligned}
$$

This proves (11).

We consider the following two cases:

1. $T$ is not continuous. Then also $U$ is not continuous. According to (2) for each $k \in \mathbf{N}$ we fix a map

$$
V_{k}: E \rightarrow E, \text { continuous }
$$


such that

$$
\left\|V_{k}(x)-U(x)\right\| \leqslant(1+(1 / k)) \varepsilon \quad \text { whenever } x \in E .
$$

Now the following holds:

Suppose $y \in E,\|y\|=1$ and $h: E \rightarrow \mathbf{R}$ is linear such that $\|h\|=h(y)=1$. Then $h(U(\lambda y))>\lambda-4 \varepsilon$ for each $\lambda \in \mathbf{R}^{+}$.

$\|h\|=1$ together with (13), (10), (7), (9) and $\|y\|=1$ shows that

$$
\begin{aligned}
h(- & \left.V_{k}\left(2^{-n} U^{-1}\left(2^{n} \lambda y\right)\right)+2^{n} \lambda y\right)<\left\|V_{k}\left(2^{-n} U^{-1}\left(2^{n} \lambda y\right)\right)-2^{n} \lambda y\right\| \\
& <\left\|U\left(2^{-n}\left(U^{-1}\left(2^{n} \lambda y\right)\right)\right)-2^{n} \lambda y\right\|+(1+(1 / k)) \varepsilon \\
& =\left\|U\left(2^{-n}\left(U^{-1}\left(2^{n} \lambda y\right)\right)\right)-U\left(U^{-1}\left(2^{n} \lambda y\right)\right)\right\|+(1+(1 / k)) \varepsilon \\
& \leqslant\left\|2^{-n} U^{-1}\left(2^{n} \lambda y\right)-U^{-1}\left(2^{n} \lambda y\right)\right\|+(2+(1 / k)) \varepsilon \\
& =\left(1-2^{-n}\right)\left\|U^{-1}\left(2^{n} \lambda y\right)\right\|+(2+(1 / k)) \varepsilon \\
& \leqslant\left(1-2^{-n}\right)\left(\left\|2^{n} \lambda y\right\|+\varepsilon\right)+(2+(1 / k)) \varepsilon \\
& =\left(2^{n}-1\right) \lambda+\left(3+(1 / k)-2^{-n}\right) \varepsilon .
\end{aligned}
$$

Because of the linearity of $h$ and $h(y)=1$ this gives

$$
h\left(V_{k}\left(2^{-n} U^{-1}\left(2^{n} \lambda y\right)\right)\right)>\lambda-\left(3+(1 / k)-2^{-n}\right) \varepsilon .
$$

We take into account (11) and the fact that $V_{k}$ and $h$ (cf. (12), (14)) are continuous and let $n \rightarrow \infty$ in the preceding equation and get

$$
h\left(V_{k}(\lambda y)\right) \geqslant \lambda-(3+(1 / k)) \varepsilon .
$$

It follows then from $\|h\|=1$ and (13) that

$$
h(U(\lambda y)) \geqslant h\left(V_{k}(\lambda y)\right)-(1+(1 / k)) \varepsilon \geqslant \lambda-(4+(2 / k)) \varepsilon .
$$

Since this is true for each $k \in \mathbf{N}$, (14) follows.

The next step is to prove

$$
\|x-U(x)\| \leqslant 5 \varepsilon \quad \text { for each } x \in E .
$$

Let $x \in E$ be chosen. Now take $y \in E,\|y\|=1$ such that $x-U(x)$ is an element of the half-ray starting from the origin and containing $y$. We choose $u \in E$ such that the half-ray through $y+u$ originating from $y$ is a halftangent of $B$, i.e.

$$
\|y+\delta u\|=1+o(\delta) \text { as } \delta \rightarrow+0
$$

(recall that $\|y\|=1$ ) and further

$$
x=\mu y+\vartheta u \quad \text { for suitable } \mu, \vartheta \in \mathbf{R}, \vartheta>0 \text {. }
$$


Then

$$
U(x)=\nu y+\vartheta u \text { for suitable } \nu \in \mathbf{R} .
$$

Denote by $L$ the subspace of $E$ generated by $u, y$. Because of the foregoing the line through $y$ and $y+u$ is a tac-line of $B$. Therefore the linear functional $k: L \rightarrow \mathbf{R}$ defined by $k(\xi y+\eta u):=\xi$ for $\xi, \eta \in \mathbf{R}$ is of norm 1 . By the theorem of Hahn and Banach it is possible to extend $k$ to a linear functional $h$ from $E$ to $\mathbf{R}$ which has also norm 1. Combining (7), $\|h\|=h(y)=1,(14)$, (18) and $h(u)=0$ yields

$$
\begin{aligned}
\|\lambda y-x\| & \geqslant\|U(\lambda y)-U(x)\|-\varepsilon \\
& >h(U(\lambda y)-U(x))-\varepsilon=h(U(\lambda y))-h(U(x))-\varepsilon \\
& \geqslant \lambda-5 \varepsilon-h(\nu y+\vartheta u)=\lambda-\nu-5 \varepsilon \text { for each } \lambda \in \mathbf{R}^{+} .
\end{aligned}
$$

On the other hand (17) and (16) imply

$$
\begin{aligned}
\|\lambda y-x\| & =\|\lambda y-\mu y-\vartheta u\|=(\lambda-\mu)\|y-(\vartheta /(\lambda-\mu)) u\| \\
& =(\lambda-\mu)(1+o(\vartheta /(\lambda-\mu)))=\lambda-\mu+o(1) \text { as } \lambda \rightarrow+\infty .
\end{aligned}
$$

Thus $\mu-\nu<5 \varepsilon$. Since $\mu-\nu=\|x-U(x)\|$ this proves (15). Since $I$ is an isometry it follows from (7) and (15) that

$$
\|T(x)-I(x)\|<5 \varepsilon \text { for each } x \in E .
$$

2. $T$ is continuous. Then one can do without the introduction of $V_{k}$ and use $U$ instead. Therefore the conclusion in (14) can be replaced by $h(U(\lambda y))>\lambda$ $-2 \varepsilon$ for each $\lambda \in \mathbf{R}^{+}$. This shows that (15) can be improved to $\|x-U(x)\|$ $<3 \varepsilon$ for each $x \in E$. Thus finally

$$
\|T(x)-I(x)\| \leqslant 3 \varepsilon \text { for each } x \in E .
$$

This proves Theorem 1 .

THEOREM 2. Let $E$ be a real normed space and let $\phi:[0,+\infty[\rightarrow[0+\infty[$ be a real function such that $\phi(\xi)=o(\xi)$ as $\xi \rightarrow+\infty$. Then there exists a real normed space $F$ with the following property: For every $\varepsilon \in \mathbf{R}^{+}$there exists an $\varepsilon$-isometry $T: E \rightarrow F$ such that there exists no isometry $I: E \rightarrow F$ for which $\| T(x)-$ $I(x) \|=o(\phi(\|x\|))$ as $\|x\| \rightarrow \infty$ uniformly.

Proof. After replacing $\phi$ by a function $>\phi$ if necessary one may assume: $\phi$ is strictly concave and continuous,

$$
\phi \text { is strictly increasing and } \lim _{\xi \rightarrow+\infty} \phi(\xi)=+\infty \text {. }
$$

After replacing $\phi$ by $\phi-\phi(0)$ one may further assume

$$
\phi(0)=0 \text {. }
$$

(19) -(21) imply 


$$
\left|\phi\left(\xi_{1}\right)-\phi\left(\xi_{2}\right)\right|<\phi\left(\left|\xi_{1}-\xi_{2}\right|\right) \text { for all } \xi_{1}, \xi_{2} \in[0,+\infty[\text {. }
$$

We will construct a norm $\psi$ in the $(\xi, \eta)$-plane with the following properties:

$$
\begin{gathered}
\psi \text { is strictly convex, } \\
\psi(\xi, \eta)=\psi( \pm \xi, \pm \eta) \text { for all } \xi, \eta \in \mathbf{R}, \\
\xi \leqslant \psi(\xi, \phi(\xi)) \leqslant 1+\xi \text { for each } \xi \in[0,+\infty[.
\end{gathered}
$$

For each $\delta \in] 0, \phi_{+}(0)[$ consider the half-ray originating from $(0,0)$ and containing $(1, \delta)$. (Here $\phi_{+}(0) \in \mathbf{R}^{+} \cup\{+\infty\}$ denotes the right-hand derivative of $\phi$ at $\xi=0$.) Because of (19), (21) and since $\phi(\xi)=o(\xi)$ as $\xi \rightarrow+\infty$ the intersection of this ray and the graph of $\phi$ consists of $(0,0)$ and exactly one further point $\left(\xi_{\delta}, \phi\left(\xi_{\delta}\right)\right)$. Define

$$
\left.p_{\delta}:=\frac{1}{1+\xi_{\delta}}\left(\xi_{\delta}, \phi\left(\xi_{\delta}\right)\right) \text { for } \delta \in\right] 0, \phi_{+}(0)\left[, p_{0}:=(1,0) .\right.
$$

$p_{\delta}$ is a continuous function of $\delta$ for $\delta \in\left[0, \phi_{+}(0)\left[\right.\right.$ and $p_{\delta} \rightarrow(0,0)$ as $\delta \rightarrow \phi_{+}(0)$. Therefore $P:=\left\{p_{\delta} \mid \delta \in\left[0, \phi_{+}(0)[\} \cup\{(0,0)\}\right.\right.$ is compact. Obviously $P \subset\{(\xi, \eta) \mid \xi \in[0,1], \quad \eta \in \mathbf{R}\}, \quad P \cap\{(1, \eta) \mid \eta \in \mathbf{R}\}=\{(1,0)\}$. Therefore there exists a compact set $C$ with center $(0,0)$ in the $(\xi, \eta)$-plane with the following properties: $C$ is strictly convex, symmetric with respect to the $\xi$ and $\eta$-axes, contained in the vertical strip $\{(\xi, \eta) \mid \xi \in[-1,1]\}$ and contains $P$. Now consider $C$ as the unit circle of a norm $\psi$. Then the properties of $C$ just mentioned imply (23)-(25). In particular the right-hand inequality in (25) can be proved in the following way: In case $\xi=0$ the inequality is obviously true because of (21). Suppose $\xi \in \mathbf{R}^{+}$. Choose $\delta \in \mathbf{R}^{+}$ such that $(1, \delta)$ is contained in the line through $(0,0)$ and $(\xi, \phi(\xi))$. Then $\xi=\xi_{\delta}$ holds. Since $p_{\delta}=(\xi, \phi(\xi)) /(1+\xi) \in C$ we have $\psi\left(p_{\delta}\right)<1$. This shows $\psi(\xi, \phi(\xi)) \leqslant 1+\xi$ as was to be proved. (24) yields

$$
\begin{array}{ll}
\psi\left(\xi, \eta_{1}\right) \leqslant \psi\left(\xi, \eta_{2}\right) & \text { whenever } \xi, \eta_{1}, \eta_{2} \in \mathbf{R},\left|\eta_{1}\right|<\left|\eta_{2}\right|, \\
\psi\left(\xi_{1}, \eta\right) \leqslant \psi\left(\xi_{2}, \eta\right) & \text { whenever } \xi_{1}, \xi_{2}, \eta \in \mathbf{R},\left|\xi_{1}\right|<\left|\xi_{2}\right| .
\end{array}
$$

Now we show

$$
\left.\left.\xi<\psi(\xi, \alpha \phi(\xi))<\alpha+\xi \text { for all } \xi \in \mathbf{R}^{+}, \alpha \in\right] 0,1\right] .
$$

Choose $\xi, \alpha$. We consider (19), (21) and $\phi\left(\xi^{\prime}\right)=o\left(\xi^{\prime}\right)$ as $\xi^{\prime} \rightarrow+\infty$. Therefore the intersection of the half-ray through $(\xi, \alpha \phi(\xi))$ originating from $(0,0)$ and the graph of $\phi$ consists of $(0,0)$ and one further point $\left(\xi^{\prime}, \phi\left(\xi^{\prime}\right)\right)$. Thus $\alpha \phi(\xi) / \xi=\phi\left(\xi^{\prime}\right) / \xi^{\prime}$. From this coupled with (25) we obtain 


$$
\begin{aligned}
\psi(\xi, \alpha \phi(\xi)) & =\xi \psi\left(1, \alpha \frac{\phi(\xi)}{\xi}\right)=\xi \psi\left(1, \frac{\phi\left(\xi^{\prime}\right)}{\xi^{\prime}}\right) \\
& =\frac{\xi}{\xi^{\prime}} \psi\left(\xi^{\prime}, \phi\left(\xi^{\prime}\right)\right)<\frac{\xi}{\xi^{\prime}}\left(1+\xi^{\prime}\right)=\alpha \frac{\phi(\xi)}{\phi\left(\xi^{\prime}\right)}+\xi<\alpha+\xi .
\end{aligned}
$$

This proves (28).

Define

$$
F:=E \oplus \mathbf{R} \text { and }\|(x, \xi)\|:=\psi(\|x\|, \xi) \quad \text { for }(x, \xi) \in F .
$$

$F$ is a real normed space. Let $\varepsilon \in \mathbf{R}^{+}$be chosen and let $T: E \rightarrow F$ be defined by

$$
T(x):=(x, \varepsilon \phi(\|x\|)) \text { for } x \in E .
$$

The next step is to show

$$
T: E \rightarrow F \text { is an } \varepsilon \text {-isometry. }
$$

Indeed, we combine (30), (29), (26), (22), (28) and have for $x, y \in E$

$$
\begin{aligned}
0 & <\|T(x)-T(y)\|-\|x-y\| \\
& =\psi(\|x-y\|, \varepsilon(\phi(\|x\|)-\phi(\|y\|)))-\|x-y\| \\
& <\psi(\|x-y\|, \varepsilon \phi(|\|x\|-\|y\||))-\|x-y\| \\
& <\psi(\|x-y\|, \varepsilon \phi(\|x-y\|))-\|x-y\|<\varepsilon+\|x-y\|-\|x-y\|=\varepsilon .
\end{aligned}
$$

Our goal is to prove the following.

Let $I: E \rightarrow F$ be an isometry. Then

$$
\|T(x)-I(x)\| \neq o(\phi(\|x\|)) \text { as }\|x\| \rightarrow \infty \text { uniformly. }
$$

Suppose $\|T(x)-I(x)\|=o(\phi(\|x\|))$. Because of $\lim _{\xi \rightarrow \infty} \phi(\xi)=+\infty$ (cf. (20)) this also holds if $I$ is replaced by $I-I(0)$. Therefore one can assume

$$
I(0)=0 \text {. }
$$

Suppose $p \in E$ with $\|p\|=1$. Let

$$
I(p)=:(q, \xi), \quad I(\lambda p)=:\left(q_{\lambda}, \xi_{\lambda}\right) \text { for } \lambda \in[1,+\infty[.
$$

Since $I$ is an isometry this combined with (29), (33) and $\|p\|=1$ yields

$$
\begin{gathered}
\psi(\|q\|, \xi)=(\|I(p)\|=\|I(p)-I(0)\|=\|p-0\|)=1, \\
\psi\left(\left\|q_{\lambda}\right\|, \xi_{\lambda}\right)=\lambda, \psi\left(\left\|q_{\lambda}-q\right\|, \xi_{\lambda}-\xi\right)=\lambda-1 \text { for } \lambda \in[1,+\infty[.
\end{gathered}
$$

Therefore (27) and the triangle inequality for $\psi$ imply

$$
\begin{aligned}
\lambda-1 & =\psi\left(\left\|q_{\lambda}-q\right\|, \xi_{\lambda}-\xi\right)>\psi\left(\left\|q_{\lambda}\right\|-\|q\|, \xi_{\lambda}-\xi\right) \\
& >\psi\left(\left\|q_{\lambda}\right\|, \xi_{\lambda}\right)-\psi(\|q\|, \xi)=\lambda-1 \text { for each } \lambda \in[1,+\infty[.
\end{aligned}
$$


Thus equality holds throughout. Since $\psi$ is strictly convex (cf. (23)) this shows $\left(\left\|q_{\lambda}\right\|, \xi_{\lambda}\right)=\mu(\|q\|, \xi)$ for suitable $\mu \in \mathbf{R}^{+}$. Taking into account that the norms of $\left(\left\|q_{\lambda}\right\|, \xi_{\lambda}\right)$ and $(\|q\|, \xi)$ are $\lambda$ and 1 resp. (cf. (35)) $\mu=\lambda$ follows. Hence

$$
\left\|q_{\lambda}\right\|=\lambda\|q\|, \quad \xi_{\lambda}=\lambda \xi
$$

Combining this with (30), (34), (29), $\|p\|=1$, (27) and $\phi(\xi)=o(\xi)$ as $\xi \rightarrow$ $+\infty$ one obtains

$$
\begin{aligned}
\|T(\lambda p)-I(\lambda p)\| & =\left\|\left(\lambda p-q_{\lambda}, \varepsilon \phi(\|\lambda p\|)-\xi_{\lambda}\right)\right\| \\
& =\psi\left(\left\|\lambda p-q_{\lambda}\right\|, \varepsilon \phi(\lambda)-\lambda \xi\right) \geqslant \psi(0, \varepsilon \phi(\lambda)-\lambda \xi) \\
& =|\varepsilon \phi(\lambda)-\lambda \xi| \psi(0,1) \neq o(\phi(\lambda)) \quad \text { as } \lambda \rightarrow \infty
\end{aligned}
$$

in contradiction to $\|T(x)-I(x)\|=o(\phi(\|x\|))$ as $\|x\| \rightarrow \infty$ uniformly. Thus (32) holds, which completes the proof of Theorem 2.

\section{The finite dimensional case.}

TheORem 3. Let $E$ and $F$ be finite dimensional real normed spaces. Suppose that $\varepsilon \in \mathbf{R}^{+}$and that $T: E \rightarrow F$ is a surjective $\varepsilon$-isometry. Then there exists an isometry $I: E \rightarrow F$ such that $\|T(x)-I(x)\|<5 \varepsilon$ for each $x \in E$. If $T$ is continuous one can choose $I$ in such a way that $\|T(x)-I(x)\|<3 \varepsilon$ whenever $x \in E$.

REMARK. In a similar way as was done for Theorem 1 it is also possible to improve Theorem 3. We omit those cases covered by the remark after Theorem 1. Let $K$ be a subset of $E$ with diam $K=2$ and denote $\operatorname{dim} E$ by $d$. If $E$ is Euclidean, a well-known theorem of Jung asserts that $K$ is contained in a ball of radius $(2 d /(d+1))^{1 / 2}$. In the general case Bohnenblust proved that $K$ is contained in a ball of radius $2 d /(d+1)$ (cf. [8, pp. 133, 134]). These results can be used to improve the constants 3,5 in Theorem 3 to $3(d /(2 d+$ $1))^{1 / 2}<212132 \ldots, 5(d /(2 d+2))^{1 / 2}<353553 \ldots$ in the Euclidean case and to $3 d /(d+1), 5 d /(d+1)$ in the general case. Other improvements are possible.

Proof. As in the proof of Theorem 1 some preliminary remarks are in order. By Theorem 2.2 of R. D. Bourgin [6] the following holds:

Let $M, N$ be finite dimensional real normed spaces, $\vartheta \in \mathbf{R}^{+}$, $S: M \rightarrow N$ a surjective $\vartheta$-isometry such that $S(0)=0$ and $k_{1}$, $k_{2}, \ldots$ a strictly increasing sequence in $\mathbf{N}$. Suppose that for some $x, y \in M$ the limits

$$
\lim _{k \rightarrow \infty} k_{n}^{-1} S\left(k_{n} x\right), \quad \lim _{n \rightarrow \infty} k_{n}^{-1} S\left(k_{n} x\right)
$$

exist (in $N$ ), then $\lim _{n \rightarrow \infty} k_{n}^{-1} S\left(k_{n}(x+y) / 2\right)$ also exists. 
Let $\delta \in \mathbf{R}^{+}$, a subset of a metric space is said to be $\delta$-onto, if for each point of the space there exists a point of the subset such that the distance between these two points is less than $\delta$. The remark after Definition 1.6 of [6] shows that

(1) holds if 'surjective' is replaced by

$$
\text { ' } S(M) \text { is } \delta \text {-onto for some } \delta \in \mathbf{R}^{+} \text {. }
$$

Now the proof of Theorem 3 runs as follows. After replacing $T$ by $T-T(0)$ if $T(0) \neq 0$ we can assume without loss of generality that

$$
T(0)=0 \text {. }
$$

Since $E$ is finite dimensional we may suppose that $E$ is endowed with ordinary Lebesgue measure. Denote by $B$ the solid unit ball of $E$ and by $C$ the minimal ellipsoid of $B$. That is, $C$ is the unique ellipsoid in $E$ containing $B$ of minimal measure (cf. Danzer, Laugwitz and Lenz [7]). Since $B$ is symmetric in 0 , this is true also for $C$. Therefore one can consider $C$ as the unit ball of some Euclidean norm || on $E$. Denote by $\langle$,$\rangle the inner product$ generated by $\| . B \subset C$ and the fact that any two norms on $E$ are equivalent imply

$$
|x| \leqslant\|x\| \leqslant \alpha|x| \text { whenever } x \in E
$$

for some fixed $\alpha \in[1,+\infty[$. We next prove that

$$
\text { bd } B \cap \text { bd } C \text { contains a basis of } E \text {. }
$$

Suppose (5) is not true. Then bd $B \cap$ bd $C$ is contained in a subspace of $E$ of codimension 1. Consider this subspace as the equator plane of $C$. Expanding $C$ slightly in a neighborhood of the equator and compressing it on the poles one can obtain an ellipsoid of smaller measure than $C$ but still containing $B$. This contradicts the minimality of $C$, thus proving (5).

$T: E \rightarrow F$ is a surjective $\varepsilon$-isometry with $T(0)=0$. Hence it is possible to choose a map $T^{-1}: F \rightarrow E$ in the following manner: $T^{-1}(0):=0$ and for each $z \in F \backslash\{0\}$ let $T^{-1}(z)$ be any point $x \in E$ for which $T(x)=z$. Obviously $T^{-1}$ has the following properties:

$$
\begin{aligned}
& T^{-1}: F \rightarrow E \text { is an } \varepsilon \text {-isometry, } T^{-1}(0)=0, \\
& \left\|T\left(T^{-1}(z)\right)-z\right\|<\varepsilon \text { for each } z \in F, \\
& \left\|T^{-1}(T(x))-x\right\|<\varepsilon \text { for each } x \in E .
\end{aligned}
$$

(In (7) one has even $T\left(T^{-1}(z)\right)=z$.)

Up to (14) we proceed along the same line as Hyers and Ulam [12], first proving 


$$
\|(T(x) / 2)-T(x / 2)\|<2^{-1 / 2} \alpha(18 \varepsilon\|x\|)^{1 / 2}+5 \alpha \varepsilon
$$

for each $x \in E \backslash\{0\}$ for which $x /\|x\| \in$ bd $B \cap$ bd $C$.

Choose $x$. By definition of || one has

$$
\|x\|=|x| \text {. }
$$

The first step in the proof of (9) is to prove that

$$
\begin{aligned}
& \text { for each } y \in E \text { such that }\|y\|<(\|x\|+5 \varepsilon) / 2,\|y-x\|< \\
& (\|x\|+5 \varepsilon) / 2 \text { it follows }\|y-(x / 2)\|<\alpha(9 \varepsilon\|x\|)^{1 / 2}+3 \alpha \varepsilon .
\end{aligned}
$$

Let $y$ be chosen. (10) and (4) imply

$$
|y| \leqslant(|x|+5 \varepsilon) / 2, \quad|y-x|<(|x|+5 \varepsilon) / 2 .
$$

Obviously

$$
\begin{gathered}
2|y-(x / 2)|^{2}=2|y|^{2}+\left(|x|^{2} / 2\right)-2\langle x, y\rangle, \\
|y-x|^{2}=|y|^{2}+|y|^{2}-2\langle x, y\rangle .
\end{gathered}
$$

Together with (12) this yields

$$
\begin{aligned}
2|y-(x / 2)|^{2} & =\left(|y|^{2}+|x|^{2}-2\langle x, y\rangle\right)+|y|^{2}-\left(|x|^{2} / 2\right) \\
& =|y-x|^{2}+|y|^{2}-\left(|x|^{2} / 2\right) \\
& <\left((|x|+5 \varepsilon)^{2} / 2\right)-\left(|x|^{2} / 2\right)<5 \varepsilon|x|+13 \varepsilon^{2} .
\end{aligned}
$$

Consequently

$$
|y-(x / 2)|^{2}< \begin{cases}9 \varepsilon|x| & \text { if }|x|>\varepsilon \\ 9 \varepsilon^{2} & \text { if }|x|<\varepsilon\end{cases}
$$

Thus in any case

$$
|y-(x / 2)|<(9 \varepsilon|x|)^{1 / 2}+3 \varepsilon .
$$

From this it follows by (4) and (10)

$$
\|y-(x / 2)\|<\alpha(9 \varepsilon\|x\|)^{1 / 2}+3 \alpha \varepsilon .
$$

This proves (11). We will apply (11) with $y:=T^{-1}(T(x) / 2)$. Combining (3) with the fact that $T$ is an $\varepsilon$-isometry we have

$$
\begin{gathered}
\|T(x) / 2\|=\|T(x)-T(0)\| / 2<(\|x-0\|+\varepsilon) / 2=(\|x\|+\varepsilon) / 2, \\
\|(T(x) / 2)-T(x)\|=\|T(x) / 2\|<(\|x\|+\varepsilon) / 2 .
\end{gathered}
$$

This together with (6) and (8) amounts to 


$$
\begin{aligned}
&\left\|T^{-1}(T(x) / 2)\right\|=\left\|T^{-1}(T(x) / 2)-T^{-1}(0)\right\| \leqslant\|(T(x) / 2)-0\|+\varepsilon \\
&<(\|x\|+3 \varepsilon) / 2 \leqslant(\|x\|+5 \varepsilon) / 2 \\
&\left\|T^{-1}(T(x) / 2)-x\right\| \leqslant\left\|T^{-1}(T(x) / 2)-T^{-1}(T(x))\right\|+\varepsilon \\
& \leqslant\|(T(x) / 2)-T(x)\|+2 \varepsilon<(\|x\|+5 \varepsilon) / 2 .
\end{aligned}
$$

Thus by (11) with $y:=T^{-1}(T(x) / 2)$

$$
\left\|T^{-1}(T(x) / 2)-(x / 2)\right\|<\alpha(9 \varepsilon\|x\|)^{1 / 2}+3 \alpha \varepsilon .
$$

The last inequality, the fact that $T$ is an $\varepsilon$-isometry, (7) and $\alpha>1$ imply

$$
\begin{aligned}
\|(T(x) / 2)-T(x / 2)\| & \leqslant \alpha(9 \varepsilon\|x\|)^{1 / 2}+3 \alpha \varepsilon+\varepsilon+\varepsilon \\
& \leqslant 2^{-1 / 2} \alpha(18 \varepsilon\|x\|)^{1 / 2}+5 \alpha \varepsilon,
\end{aligned}
$$

confirming (9).

We now show by induction the following proposition:

Let $n \in \mathbf{N}$. Then for each $x \in E \backslash\{0\}$ for which $x /\|x\| \in$ bd $B \cap$ bd $C$ we have

$$
\begin{aligned}
& \left\|T\left(2^{-n} x\right)-2^{-n} T(x)\right\| \\
& \quad \leqslant 2^{-n / 2} \alpha(18 \varepsilon\|x\|)^{1 / 2} \sum_{0}^{n-1} 2^{-k / 2}+\left(1-2^{-n}\right) 10 \alpha \varepsilon \\
& \\
& \quad\left(<2^{-n / 2} \beta\|x\|^{1 / 2}+\gamma, \beta:=\alpha(18 \varepsilon)^{1 / 2} \sum_{0}^{\infty} 2^{-k / 2}, \gamma:=10 \alpha \varepsilon\right) .
\end{aligned}
$$

For $n=1$ (13) and (9) agree. Hence (13) is true in this case. Let $n \in \mathbf{N}$ and suppose that (13) is true for $n$. We consider $n+1$. Suppose $x \in E \backslash\{0\}$ such that $x /\|x\| \in$ bd $B \cap$ bd $C$. Then $x / 2 \in E \backslash\{0\}$ and $(x / 2) /\|(x / 2)\|=$ $x /\|x\| \in$ bd $B \cap$ bd $C$. Therefore by (13)

$$
\begin{aligned}
& \left\|T\left(2^{-(n+1)} x\right)-2^{-n} T(x / 2)\right\| \\
& \quad<2^{-(n+1) / 2} \alpha(18 \varepsilon\|x\|)^{1 / 2} \sum_{0}^{n-1} 2^{-k / 2}+\left(1-2^{-n}\right) 10 \alpha \varepsilon .
\end{aligned}
$$

Combining this with (9) yields 


$$
\begin{aligned}
& \left\|T\left(2^{-(n+1)} x\right)-2^{-(n+1)} T(x)\right\| \\
& \leqslant\left\|T\left(2^{-(n+1)} x\right)-2^{-n} T(x / 2)\right\|+2^{-n}\|T(x / 2)-(T(x) / 2)\| \\
& <2^{-(n+1) / 2} \alpha(18 \varepsilon\|x\|)^{1 / 2} \sum_{0}^{n-1} 2^{-k / 2}+\left(1-2^{-n}\right) 10 \alpha \varepsilon \\
& \quad+2^{-n / 2} 2^{-1 / 2} \alpha(18 \varepsilon\|x\|)^{1 / 2} 2^{-n / 2}+2^{-n}\left(1-2^{-1}\right) 10 \alpha \varepsilon \\
& =2^{-(n+1) / 2} \alpha(18 \varepsilon\|x\|)^{1 / 2} \sum_{0}^{n} 2^{-k / 2}+\left(1-2^{-(n+1)}\right) 10 \alpha \varepsilon .
\end{aligned}
$$

This completes the inductive proof of (13).

We now show

$$
\begin{aligned}
& \lim _{n \rightarrow \infty} 2^{-n} T\left(2^{n} x\right) \text { exists (in } F \text { ) for each } x \in E \text { such that } \\
& x=0 \text { or } x \neq 0 \text { and } x /\|x\| \in \text { bd } B \cap \text { bd } C \text {. }
\end{aligned}
$$

Choose $x$. In case $x=0$ the assertion of (14) is obviously true. Suppose $x \neq 0$. We substitute $m$ for $n$ and $2^{l+m} x$ for $x$ in (13), multiply by $2^{-l}$ and obtain

$$
\left\|2^{-l} T\left(2^{l} x\right)-2^{-(l+m)} T\left(2^{-(l+m)} x\right)\right\| \leqslant 2^{-l / 2} \beta\|x\|^{1 / 2}+2^{-l} \gamma
$$

whenever $l, m \in \mathbf{N}$. Hence $\left(2^{-n} T\left(2^{n} x\right)\right)$ is a Cauchy sequence. This proves (14). The following statement improves upon (14):

$$
\lim _{n \rightarrow \infty} 2^{-n} T\left(2^{n} x\right) \text { exists for each } x \in E \text {. }
$$

Because of (5) there exists a basis $b_{1}, \ldots, b_{d} \in$ bd $B \cap$ bd $C$ of $E$. Provided that $x$ is a multiple of some $b_{i}, \lim _{n \rightarrow \infty} 2^{-n} T\left(2^{n} x\right)$ exists by (14). Any element of the subspace of $E$ generated by $b_{1}, b_{2}$ is the arithmetic mean of suitable multiples of $b_{1}$ and $b_{2}$. Any element of the subspace generated by $b_{1}, b_{2}, b_{3}$ is the arithmetic mean of an element of the subspace generated by $b_{1}, b_{2}$ and a multiple of $b_{3}$ and so on. Coupling this with (1) yields (15).

Let $I: E \rightarrow F$ be defined by

$$
I(x):=\lim _{n \rightarrow \infty} 2^{-n} T\left(2^{n} x\right) \text { for each } x \in E .
$$

(3) implies

$$
I(0)=0
$$

The next step is to show that

$$
I \text { is an isometry. }
$$

Indeed, let $x, y \in E$ be chosen. Since $T$ is an $\varepsilon$-isometry, we have

$$
\left|\left\|T\left(2^{n} x\right)-T\left(2^{n} y\right)\right\|-\left\|2^{n} x-2^{n} y\right\|\right|<\varepsilon \quad \text { whenever } n \in \mathbf{N} \text {. }
$$

Multiply this by $2^{-n}$. Now (16) shows $\|I(x)-I(y)\|=\|x-y\|$. This proves (18). 
Since $I: E \rightarrow F$ is an isometry and hence a homeomorphism of $E$ onto $I(E) \subset F$ it follows

$$
\operatorname{dim} E<\operatorname{dim} F .
$$

To prove the opposite inequality

$$
\operatorname{dim} F<\operatorname{dim} E
$$

we proceed as follows: In our proof of Theorem 3 we used so far only the following properties of $T: T(0)=0, T: E \rightarrow F$ is an $\varepsilon$-isometry and $T$ is surjective. The surjectivity was necessary only for the proof of (6), (7), (8) and for the application of (1). Consider $T^{-1} . T^{-1}(0)=0$ and $T^{-1}: F \rightarrow E$ is an $\varepsilon$-isometry (see (6)). (6), (7), (8) hold if we replace $T, T^{-1}, E, F$ by $T^{-1}, T, F$, $E$. Because of (8) $T^{-1}(F) \subset E$ is $\varepsilon$-onto. This together with (2) shows that one can apply (1) for $T^{-1}$. Therefore it is possible to carry the whole proof up to (19) with $T^{-1}, F, E$ in place of $T, E, F$. Then instead of (19) the inequality (20) holds. This proves (20). (19) and (20) together show that

$$
\operatorname{dim} E=\operatorname{dim} F \text {. }
$$

We now assert

$$
I \text { is surjective and linear. }
$$

Since $I$ is an isometry, $I(E)$ is a closed subset of $F$. Since $E$ and $F$ are finite dimensional and (21) holds the theorem of Brouwer on invariance of domain (cf. [1, p. 396]) implies that $I(E)$ is an open subset of $F$. Since $F$ is the only nonempty subset of $F$ which is closed and open it follows that $I(E)=F$. Thus $I$ is surjective. $I: E \rightarrow F$ is an isometry (cf. (18)). Therefore the theorem of Mazur and Ulam (cf. [2, p. 160] and [9, p. 142]) yields the linearity of $I$. This completes the proof of (22).

$T: E \rightarrow F$ is a surjective $\varepsilon$-isometry with $T(0)=0 . I$ is a surjective linear isometry (see (18) and (22)) with $I(0)=0$ (see (17)) and $I(x)=$ $\lim _{n \rightarrow \infty} 2^{-n} T\left(2^{n} x\right)$ whenever $x \in E$ (see (16)). These are precisely the properties which are used in the proof of Theorem 1. (Compare the remark between (6) and (7) of that proof.) Therefore the proof of Theorem 1 beginning with (7) can be transferred to the present situation. Thus $\| T(x)-$ $I(x) \|<5 \varepsilon$ for each $x \in E$ and $\|T(x)-I(x)\|<3 \varepsilon$ for each $x \in E$ if in addition $T$ is continuous. This completes the proof of Theorem 3.

4. Acknowledgement. This paper was written in April 1977 during a stay at the Mathematical Institute of the Hungarian Academy of Sciences in Budapest for which the author expresses his gratitude to Professor L. Fejes Tóth. Many thanks are due to Professor A. Pelczyński for pointing out to me the paper of T. Figiel which proved to be a most valuable device for the proof of Theorem 1 above. Finally I should like to thank Professor E. Lukacs for his help in the preparation of the manuscript. 


\section{REFERENCES}

1. P. Alexandroff and H. Hopf, Topologie I, Die Grundlehren der math. Wissenschaften, vol. 45, Springer-Verlag, Berlin, 1935.

2. St. Banach, Théorie des opérations linéaires, Monografie Mat., PWN, Warsaw, 1932; reprint, Chelsea, New York, 1955.

3. D. G. Bourgin, Approximate isometries, Bull. Amer. Math. Soc. 52 (1946), 704-714.

4. Approximately isometric and multiplicative transformations on continuous function rings, Duke Math. J. 16 (1949), 385-397.

5. Classes of transformations and bordering transformations, Bull. Amer. Math. Soc. 57 (1951), 223-237.

6. __ Approximate isometries on finite dimensional Banach spaces, Trans. Amer. Math. Soc. 207 (1975), 309-328.

7. L. Danzer, D. Laugwitz and H. Lenz, Über das Löwnersche Ellipsoid und sein Analogon unter den einem konvexen Körper einbeschriebenen Ellipsoiden, Arch. Math. 8 (1957), 214-219.

8. L. Danzer, B. Grünbaum and V. Klee, Helly's theorem and its relatives, in: Convexity, Proc. Sympos. Pure Math., vol. 7, Amer. Math. Soc., Providence, R. I., 1963, pp. 101-180.

9. M. M. Day, Normed linear spaces, Ergebnisse der Math. und ihrer Grenzgebiete, vol. 21, Springer-Verlag, Berlin and New York, 1973.

10. T. Figiel, On non linear isometric embedding of normed linear spaces, Bull. Acad. Polon. Sci. Sér. Sci. Math. Astronom. Phys. 16 (1968), 185-188.

11. D. G. de Figueiredo and L. A. Karlowitz, The extension of contractions and the intersection of balls in Banach spaces, J. Functional Analysis 11 (1972), 168-178.

12. D. H. Hyers and S. M. Ulam, On approximate isometries, Bull. Amer. Math. Soc. 51 (1945), 288-292.

13. On approximate isometries on the space of continuous functions, Ann. of Math. (2) 48 (1947), 285-289.

14. E. Lukacs, Über die Stabilität einer Charakterisierung der Normalverteilung, S.-B. Österr. Akad. Wiss. Math.-Natur. K1. 185 (1976), 167-179.

15. S. M. Ulam, A collection of mathematical problems, Interscience Tract 8 (= Problems in Modern Mathematics, Science Edition), Interscience, New York, 1960 (1964).

Institut für ANAlysis, Techisische Universität Wien, A-1040 Vienna; Austria 